\title{
Regulation of cellular senescence by the essential caveolar component PTRF/Cavin-1
}

\author{
Lin Bai ${ }^{1}$, Xiaoli Deng ${ }^{1}$, Juanjuan $\mathrm{Li}^{1}$, Miao Wang ${ }^{1}$, Qian $\mathrm{Li}^{1}$, Wei An ${ }^{1}$, Deli A ${ }^{1}$, Yu-Sheng Cong ${ }^{1}$ \\ ${ }^{I}$ Key Lab of Cell Proliferation and Regulation Biology of the Ministry of Education, Institute of Cell Biology, Beijing Normal Uni- \\ versity, 19 Xin Jie Kou Wai Avenue, Beijing 100875, China
}

Polymerase I and transcript release factor (PTRF, also known as Cavin-1) is an essential component in the biogenesis and function of caveolae. Here, we show that PTRF expression is increased in senescent human fibroblasts. Importantly, overexpression of PTRF induced features characteristic of cellular senescence, whereas reduced PTRF expression extended the cellular replicative lifespan. Interestingly, we found that PTRF localized primarily to the nuclei of young and quiescent WI-38 human fibroblasts, but translocated to the cytosol and plasma membrane during cellular senescence. Furthermore, electron microscopic analysis demonstrated an increased number of caveolar structures in senescent and PTRF-transfected WI-38 cells. Our data suggest that the role of PTRF in cellular senescence is dependent on its targeting to caveolae and its interaction with caveolin-1, which appeared to be regulated by the phosphorylation of PTRF. Taken together, our findings identify PTRF as a novel regulator of cellular senescence that acts through the $\mathrm{p53} / \mathrm{p} 21$ and caveolar pathways.

Keywords: PTRF; caveolae; caveolin-1; cellular senescence

Cell Research (2011) 21:1088-1101. doi:10.1038/cr.2011.56; published online 29 March 2011

\section{Introduction}

Cellular senescence was originally described as a process of permanent cell cycle arrest after exhaustion of the replicative potential of cultured human fibroblasts [1]. Unlike quiescence, a transient growth arrest that is readily reversed by stimulation with mitogens, cellular senescence is an essentially irreversible form of cell cycle arrest and can be classified into two categories: replicative senescence due to telomere attrition and premature senescence caused by intrinsic and/or extrinsic stress factors, including activated oncogenes, DNA damage, oxidative stress, and suboptimal culture conditions [2]. Replicative senescence and premature senescence exhibit similar phenotypes characterized by an enlarged and flattened cell morphology, distinct nuclear structures of heterochromatin, activated DNA damage responses, altered gene expression profiles and metabolism, and in-

\footnotetext{
Correspondence: Yu-Sheng Cong

Tel: +86-10-5880-2030

E-mail: yscong@bnu.edu.cn

Received 12 July 2010; revised 15 October 2010; accepted 3 December 2010; published online 29 March 2011
}

duction of senescence-associated $\beta$-galactosidase (SA- $\beta$ gal) activity [3,4].

Cellular senescence has been proposed to recapitulate the aspects of organismal aging [5]. Indeed, the number of senescent cells increases with age, and senescence phenotypes can be observed with organismal aging and are considered to be predictive features of age-related pathologies and longevity in humans $[6,7]$. Senescence has also been proposed to be a tumor suppression mechanism $[8,9]$. Recent studies have demonstrated that oncogeneinduced senescence occurs in benign human and mouse tumors, but not in advanced tumors, which suggests that senescence is an initial barrier restricting cancer development [10-13]. Significantly, the restoration of p53 activity causes tumor cells to undergo cellular senescence or apoptosis, and leads to the regression of several different types of tumors in mice [14-16]. These observations support the hypothesis that senescence plays a critical role in tumor suppression in vivo by preventing malignant transformation of benign lesions and that aging and cancer may share a common biology [17]. Cellular senescence is mainly controlled by the p53-p21 and p16-pRb tumor suppressor pathways; however, upstream regulators and downstream effectors that sense and execute the telom- 
ere-based replicative senescence and telomere-independent premature senescence programs remain unclear.

Caveolae are specialized invaginations of the plasma membrane that are implicated in diverse cellular functions including signal transduction, lipid regulation and endocytosis [18]. The major structural components of caveolae consist of the caveolin family (caveolin-1, caveolin-2 and caveolin-3) and the Cavin family (PTRF/ Cavin-1, SDPR/Cavin-2, SRBC/Cavin-3 and MURC/ Cavin-4) [19-22]. PTRF was originally identified as a polymerase I and transcript release factor. It interacts with TTF-1, Pol I and the 3' end of pre-rRNA, and enhances ribosomal RNA synthesis by dissociating the ternary complex of RNA polymerase I [23]. Recently, it was demonstrated that PTRF is an essential component in the biogenesis and function of caveolae [24]. Mice that are deficient in PTRF exhibit a global loss of caveolae, dyslipidemia and glucose intolerance [25], and human PTRF mutations have been recently associated with generalized lipodystrophy [26, 27]. These observations underscore the physiological importance of PTRF.

Using a quantitative proteomic approach, we have previously shown that PTRF is upregulated in human fibroblasts undergoing both replicative and premature senescence compared to their young and quiescent counterparts [28]. In this study, we identified PTRF as a novel regulator of cellular senescence that acts through the p53/p21 and caveolar pathways.

\section{Results}

\section{Upregulation of PTRF in senescent human fibroblasts}

Previously, we used a quantitative proteomic approach to show that PTRF is differentially expressed in young replicating and senescent WI-38 cells [28]. To further characterize PTRF in cellular senescence, WI-38 cell populations at different growth stages were prepared as described previously [28]. The levels of PTRF expression, along with those of other senescence-associated proteins, were examined by western blot analysis. Consistent with the proteomic data, PTRF was specifically upregulated in senescent WI-38 cells, whereas HSP90 and collagen type I were downregulated in senescent cells, compared to young replicating or transiently growth-arrested quiescent cells (Figure 1A), which suggests that the expression of these genes is associated with cellular senescence. Consistent with a previous report [29], caveolin-1 protein levels were increased in both senescent WI-38 cells and quiescent cells (Figure 1A). We also analyzed the expression of SDPR and SRBC, the other two members of the Cavin family, in young replicating, senescent and quiescent WI-38 cells. Both
SDPR and SRBC were upregulated in quiescent cells, but SRBC was also upregulated in senescent cells (data not shown). PTRF expression at the mRNA and proteins levels was further analyzed in WI-38 and IMR-90 cells. As shown in Figure 1B, the levels of PTRF protein were increased in both senescent WI-38 and IMR-90 cells, but no apparent differences were observed in PTRF mRNA levels between young and senescent cells. These results suggest that increased expression of PTRF in senescent human fibroblasts may be due to post-translational modification(s).

\section{Regulation of cellular senescence by PTRF}

To investigate the potential role of PTRF in cellular senescence, we stably transfected a PTRF expression construct or control vector into WI-38 cells. Overexpression of PTRF significantly suppressed WI-38 cell growth (Supplementary information, Figure S1A), an effect that was associated with phenotypes that are characteristic of cellular senescence, including increased DNA damage foci of histone H2AX phosphorylation (termed $\gamma$-H2A.X) (Figure 2A, see Supplementary information, Figure S1B for a lower magnification image), increased SA- $\beta$-gal expression (Figure 2B), and a reduced replicative lifespan (Figure 2C). PTRF is an essential component in the formation and function of caveolae [24, 30], which are important in the regulation of various signaling events $[18,31,32]$. Thus, we examined the effects of PTRF overexpression on p53, p21 and pRb, which are known regulators of cellular senescence, and its effects on the signaling molecules downstream of caveolae, such as ERK1/2 and cyclin D1. Transient overexpression of PTRF resulted in increased levels of caveolin-1, p53 and p21 expression, but expression levels of $\mathrm{pRb}$ remained unchanged. In addition, overexpression of PTRF resulted in decreased expression of phosphorylated ERK1/2 and cyclin D1 (Figure 2D).

To investigate the effects of PTRF downregulation on cellular senescence, we constructed two shRNA retroviruses targeting PTRF (shPTRF1 and shPTRF2). Western blot analysis of PTRF expression in WI-38 cells that were stably transduced with shPTRF1 and shPTRF2 showed that shPTRF2 efficiently reduced PTRF expression, whereas shPTRF1 only modestly downregulated PTRF (data not shown). Subsequent experiments were performed using WI-38/shPTRF2 stable cell line. Downregulation of PTRF expression by shPTRF2 increased the proliferation potential of WI-38 cells (Supplementary information, Figure S1C). Furthermore, PTRF downregulation resulted in reduced numbers of DNA damage foci of $\gamma$-H2A.X (Figure 3A, see Supplementary information, Figure S1D for a lower magnification image) and 
A
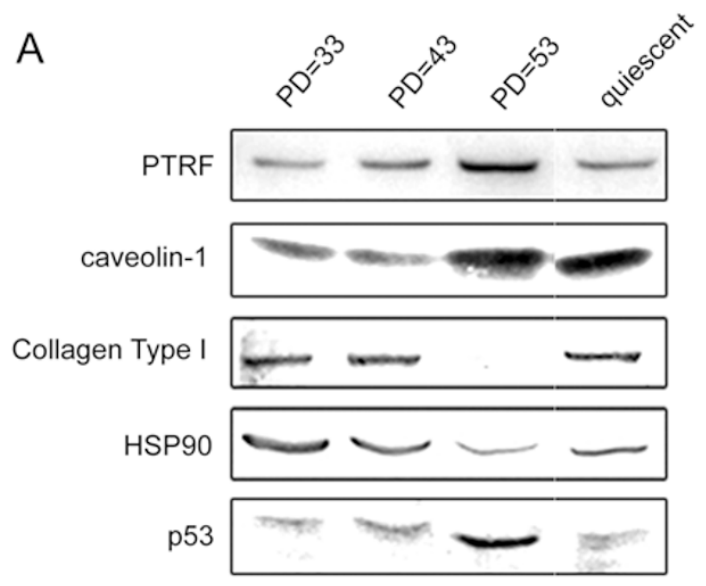

$\beta$-actin $\square$

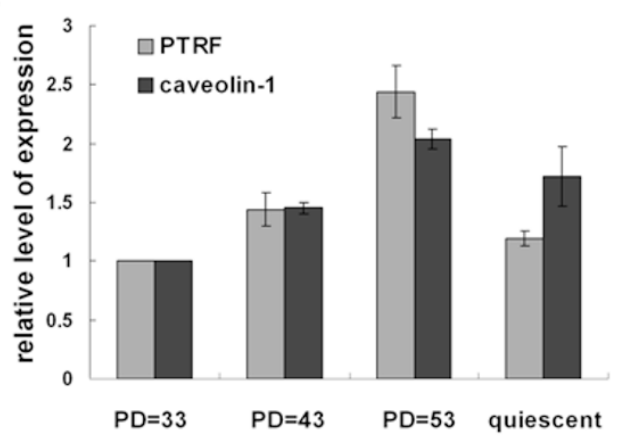

B
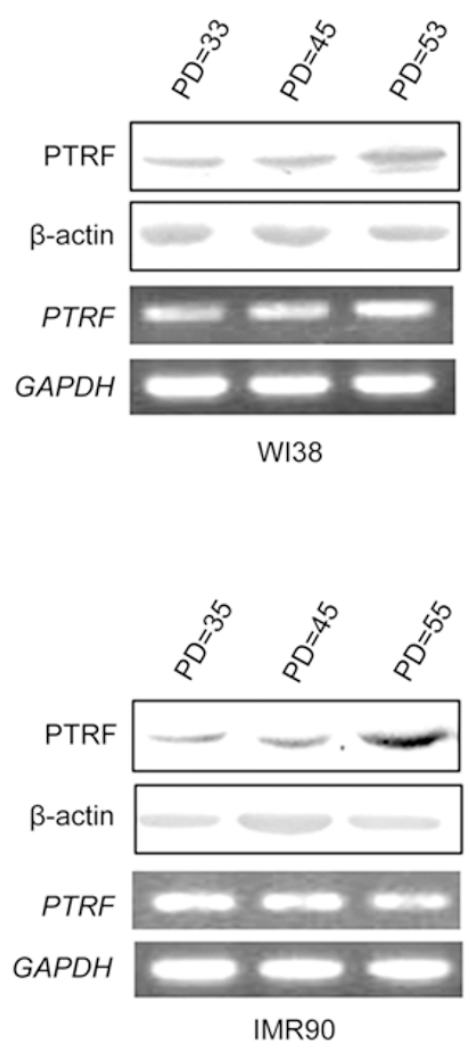

Figure 1 Upregulation of PTRF in senescent human fibroblasts. (A) Western blot analysis of PTRF and other senescenceassociated proteins in young replicating, middle-aged replicating, replicatively senescent and young serum-starved quiescent WI-38 fibroblasts. The relative expression of PTRF and caveolin-1 is shown. (B) The expression of PTRF at the mRNA and protein levels during replicative senescence of WI-38 and IMR-90 fibroblasts was assayed by RT-PCR and western blot analysis, respectively. GAPDH and $\beta$-actin were used as loading controls.

reduced expression of SA- $\beta$-gal (Figure 3B), along with extended cellular replicative lifespan of WI-38 cells (Figure $3 \mathrm{C}$ ). In contrast to overexpression of PTRF, downregulation of PTRF expression by shPTRF2 resulted in decreased expression of caveolin-1, p53 and p21, but increased phosphorylation of ERK1/2 and elevated cyclin D1 expression (Figure 3D).

To confirm that the effects of shPTRF2 on the regulation of senescence were specifically due to downregulation of PTRF, we transfected the PTRF expression construct pcDNA3.1-PTRF into the WI-38/shPTRF2 and WI-38/shcon stable cell lines. Given that shPTRF2 targeted the 3'-untranslated region of PTRF mRNA, outside of the coding region, which is the shPTRF2 target is not present in the PTRF expression construct. Thus, ectopic expression of PTRF by pcDNA3.1-PTRF should not be affected by shPTRF2 (Figure 4B). Ectopic expression of PTRF reversed the extended lifespan induced by
shPTRF2 (Figure 4A) and rapidly induced senescence in both WI-38/shPTRF and WI-38/shcon cell lines. In addition, it increased the expression of p21 and p53 (Figure 4B) and the number of SA- $\beta$-gal-positive cells (Figure 4C). These results confirm that the observed effects of shPTRF2 on the regulation of senescence were specifically due to the downregulation of PTRF. Taken together, these data show that overexpression of PTRF induces senescence, whereas the downregulation of PTRF extends cellular lifespan, and these effects are associated with the expression of senescence regulators and caveolar signaling molecules such as p53, p21, ERK and cyclin D1. These findings suggest that PTRF is a novel regulator of cellular senescence that acts via the p53/p21 and caveolar pathways.

Telomere maintenance is a critical factor in cellular senescence, and ectopic expression of the human telomerase reverse transcriptase hTERT prevents senescence in 
A
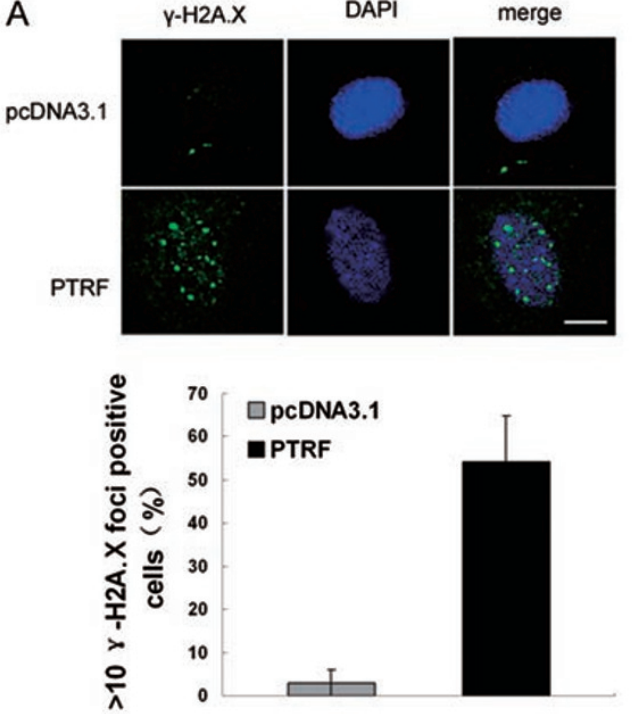

C

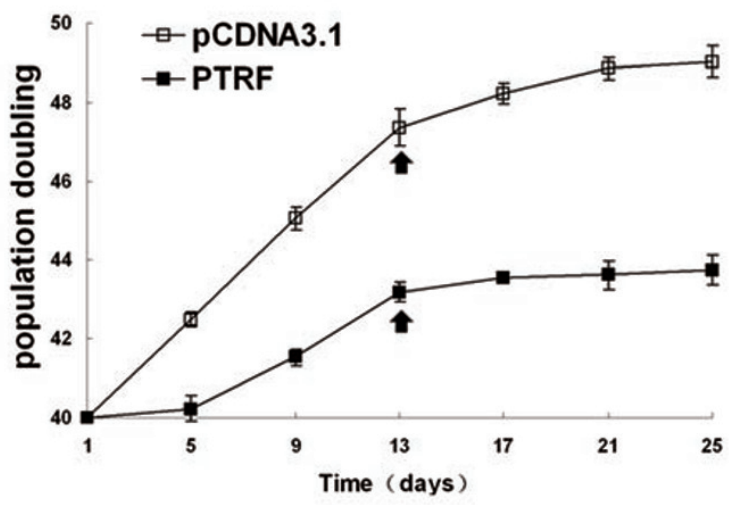

B

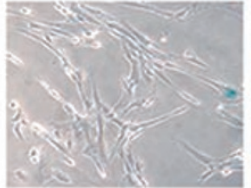

pCDNA3.1

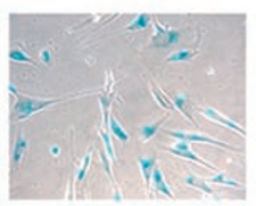

PTRF

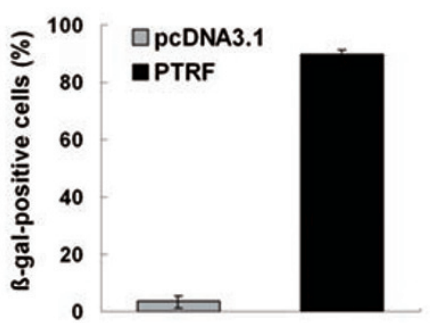

D

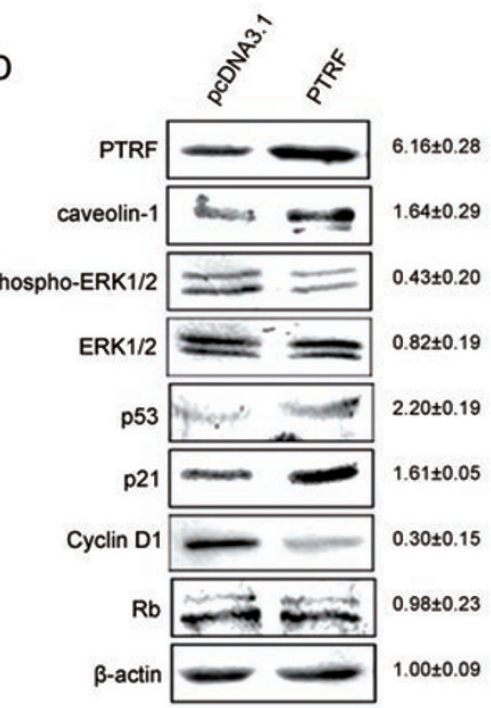

Figure 2 Overexpression of PTRF induces phenotypes characteristic of cellular senescence. (A) Accumulation of $\gamma$-H2A.X induced by overexpression of PTRF. Cells were fixed and stained with antibodies against $\gamma-\mathrm{H} 2 \mathrm{~A} . \mathrm{X}$ (green) and DAPI (blue). The $\gamma-\mathrm{H} 2 \mathrm{~A}$.X staining showed DNA foci in the nuclei of pcDNA3.1-PTRF cells, which is characteristic of senescent cells. DAPI was used to stain the nuclei. The scale bar represents $10 \mu \mathrm{m}$. Quantification of cells with $>10 \gamma-\mathrm{H} 2 \mathrm{~A}$.X is shown in the lower panel of (A). At least 200 cells were analyzed per experiment. A lower magnification image is shown in Supplementary information, Figure S1B. (B) SA- $\beta$-gal staining of senescent cells induced by overexpression of PTRF. The percentage of SA- $\beta$-gal-positive cells was calculated from three randomly chosen fields. The experiment was repeated three times, and the results are presented as mean \pm s.d. (C) Growth curves of WI-38 cells that were stably transfected with pcDNA3.1 or pcDNA3.1-PTRF. Cumulative population doubling (PD) was calculated at each time point. The experiment was repeated three times, and the data shown represent the mean \pm s.d. The arrows marked under the curve indicate the time points at which the $\gamma-\mathrm{H} 2 \mathrm{~A}$.X staining assay (A) and the SA- $\beta$-gal staining assay (B) were performed. (D) Western blot analysis of PTRF, senescence-associated proteins and caveolae-related proteins in WI-38 cells that were transiently transfected with the pcDNA3.1 control vector, or pcDNA3.1-PTRF. A representative blot is shown. Fold changes of the protein levels in pcDNA3.1PTRF-transfected cells compared to pcDNA3.1-transfected cells were determined densitometrically as the ratio (mean \pm s.d.) from three independent experiments, and are indicated at the right side of the blot. Expression level of each given protein in pcDNA3.1-transfected cells was arbitrarily set at 1. $\beta$-actin was used for normalization.

normal human fibroblasts by maintaining their telomeres [33]. To investigate whether the effects of PTRF on cel- lular senescence involve telomere maintenance, hTERTimmortalized WI-38/hTERT cells and control WI-38/ 
GFP cells [34] were transiently transfected with the PTRF expression construct pcDNA3.1-PTRF or the pcDNA3.1 control vector. Overexpression of PTRF induced cellular senescence in both cell lines WI-38/hTERT and WI-38/GFP, along with an upregulation of p21 and p53 expression and an increase in the number of SA- $\beta$-gal-
A

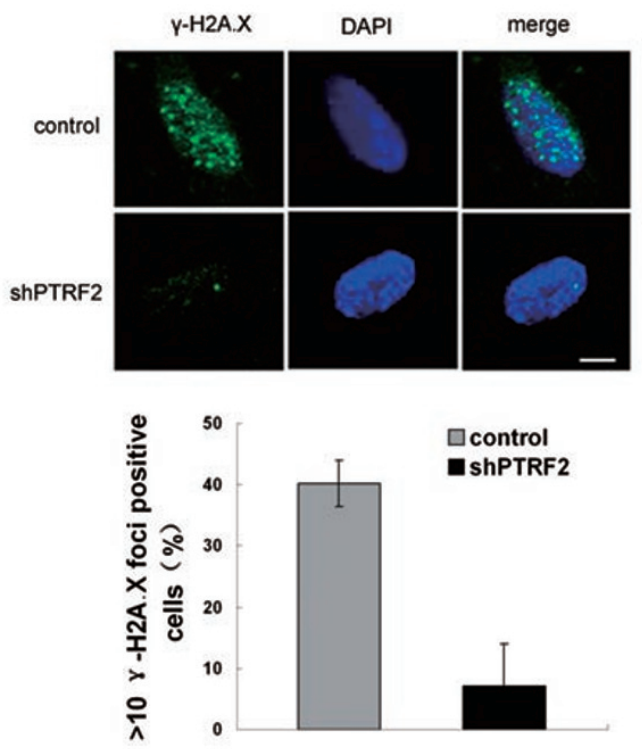

C

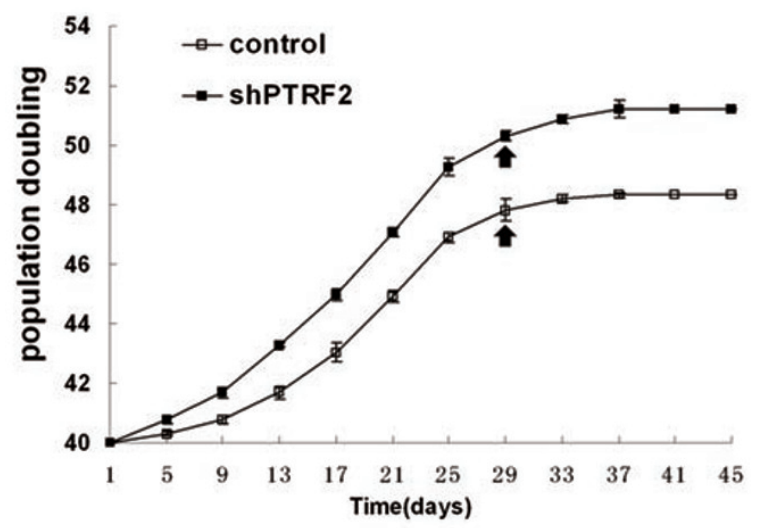

B
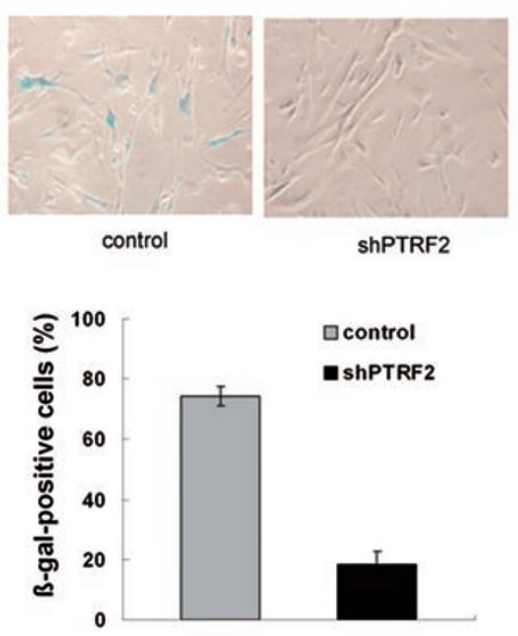

D

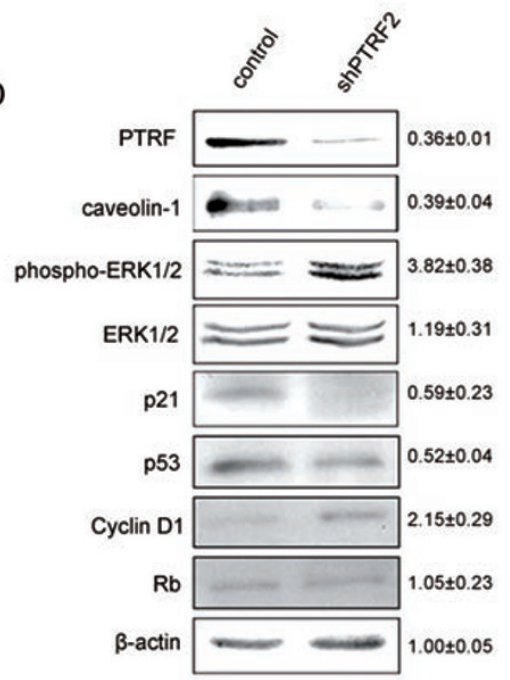

Figure 3 shRNA-mediated knockdown of PTRF results in lifespan extension. WI-38 cells were stably transduced with the control retroviral or the shPTRF retroviral constructs. (A) Reduction of $\gamma-\mathrm{H} 2 \mathrm{~A}$.X staining induced by shRNA-mediated knockdown of PTRF. Cells were fixed and stained with antibodies against $\gamma-\mathrm{H} 2 \mathrm{~A}$.X (green) and DAPI (blue). DAPI was added to stain the nuclei. The scale bar represents $10 \mu \mathrm{m}$. Quantification of cells with $>10 \gamma-\mathrm{H} 2 \mathrm{~A}$.X is shown in the lower panel of (A). At least 200 cells were analyzed per experiment. A lower magnification image is shown in Supplementary information, Figure S1D. (B) SA- $\beta$-gal staining of senescent WI-38 cells transduced with the indicated shRNA. The percentage of cells with SA$\beta$-gal-positive cells was calculated from three randomly chosen fields. The experiment was repeated three times, and the results represent the mean \pm s.d. (C) Growth curves of WI-38 cells that were stably transfected with the indicated shRNA. Cumulative population doubling (PD) was calculated at each time point. The experiment was repeated three times, and data represent the mean \pm s.d. The arrows marked under the curve indicate the time points at which $\gamma-H 2 A . X$ staining (A) and SA$\beta$-gal staining (B) were performed. (D) Western blot analysis of PTRF, senescence-associated proteins and caveolae-related proteins in control shRNA-transduced and shPTRF-transduced WI-38 cells. A representative blot is shown. Fold changes of the protein levels in shPTRF-transduced cells compared to control shRNA-transduced cells were determined densitometrically as the ratio (mean \pm s.d) from three experiments and are indicated at the right side of the blot. The expression level of each given protein expression in control shRNA-transduced cells was arbitrarily set at 1 . $\beta$-actin was used for normalization. 
A

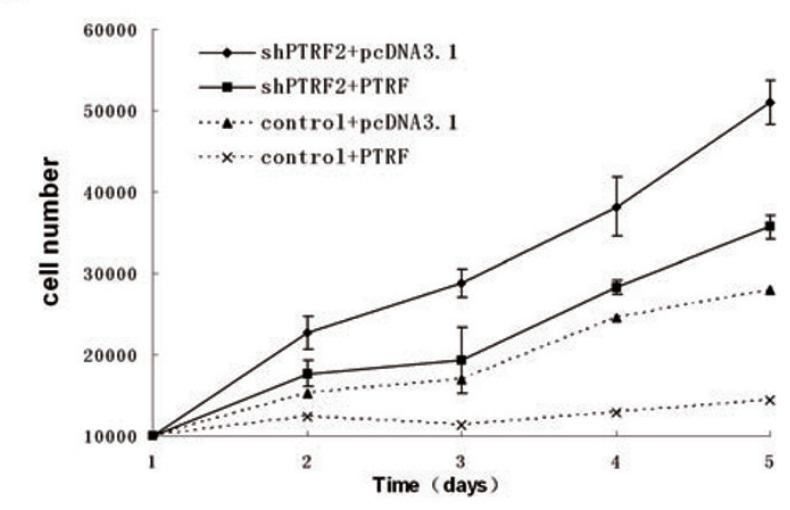

C
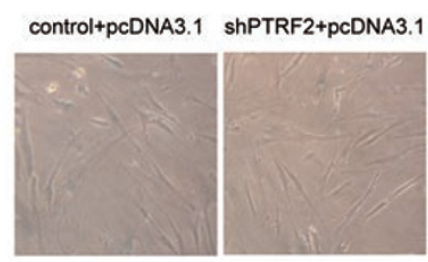

control+PTRF

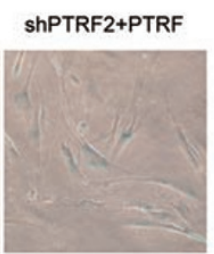

B

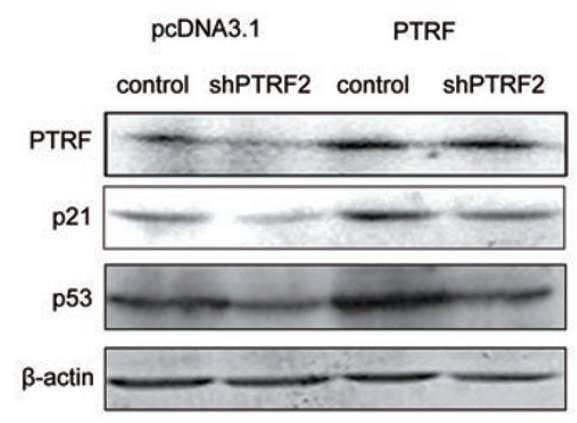

Figure 4 (A) Ectopic expression of PTRF reverses shPTFR-mediated lifespan extension in WI-38 cells. The WI-38-shPTRF2 stable cell line $(P D=43)$ was transiently transfected with pcDNA3.1 or pcDNA3.1-PTRF. Growth curves were generated by cell number at the indicated times. The data presented are from three independent experiments. (B) Western blot analysis of the cell lines in (A) to detect PTRF, p53 and p21 expression levels. $\beta$-actin was used as a loading control. (C) SA- $\beta$-gal staining of the cells indicated in (A). The percentage of SA- $\beta$-gal-positive cells was calculated from three randomly chosen fields. The experiment was repeated three times, and the results represent the mean \pm s.d.

positive cells, regardless of hTERT expression (Supplementary information, Figure S2). These results indicate that the effects of PTRF on cellular senescence in WI-38 cells are independent of telomere maintenance.

\section{Differential subcellular distribution of PTRF during cel- lular senescence}

PTRF was originally identified as a nuclear protein involved in ribosomal RNA synthesis [23] and was recently found to be a critical player in the biogenesis and function of caveolae in the plasma membrane [24, 30]. Thus, we examined the subcellular distribution of PTRF during cellular senescence by immunofluorescent staining. The specificity of the PTRF antibody in immunofluorescent staining was tested using shPTRF knockdown cells, which demonstrated attenuated PTRF fluorescent signals compared to control shRNA cells, indicating that the PTRF antibody was specific in immunofluorescent staining (Supplementary information, Figure S3). Interestingly, PTRF was localized primarily to the nuclei of young and quiescent WI-38 cells, whereas it was mainly present in the cytosol and plasma membrane in senescent WI-38 cells, in which it colocalized with caveolin-1 (Fig- ure 5A). Consistent with these observations, western blot analysis of subcellular fractions showed that PTRF was present primarily in the nuclear fraction of young WI-38 cells, whereas it was enriched in the membrane fraction of senescent WI-38 cells (Figure 5B). To further confirm this differential subcellular distribution in young and senescent cells, we performed co-immunoprecipitation assays to assess the interaction of PTRF with caveolin-1, a structural component of caveolae. Immunoprecipitation assays were conducted with cell lysates from young and senescent WI-38 cells that contained an equivalent amount of PTRF (Figure 5C). Consistent with the results of the immunofluorescent staining assays (Figure 5A), PTRF and caveolin-1 co-immunoprecipitated in senescent WI-38 cells, but did so less efficiently in young WI38 cells (Figure 5C). These results show that the subcellular distribution of PTRF is regulated during cellular senescence.

\section{Increased numbers of caveolar structures during cellular} senescence

Given that PTRF is upregulated and translocates to the plasma membrane in senescent fibroblasts (Figures 
1 and 5), it is conceivable that PTRF may promote the formation of caveolae during cellular senescence. Thus, we examined the number of caveolar structures in young replicating and senescent WI-38 cells and in
PTRF-transfected WI-38 cells by electron microscopic analysis. Consistent with the previous study showing that senescent cells have an increased number of caveolar structures compared to young replicating cells [29],

A

PTRF

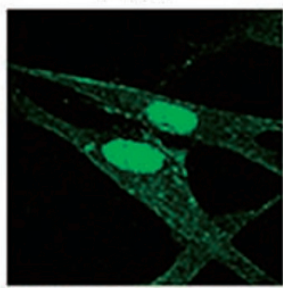

$\mathrm{PD}=33$

$\mathrm{PD}=43$
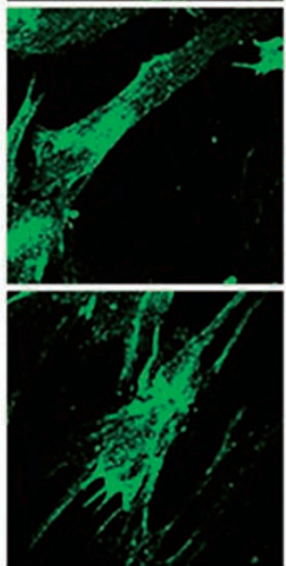

$\mathrm{PD}=53$

quiescent
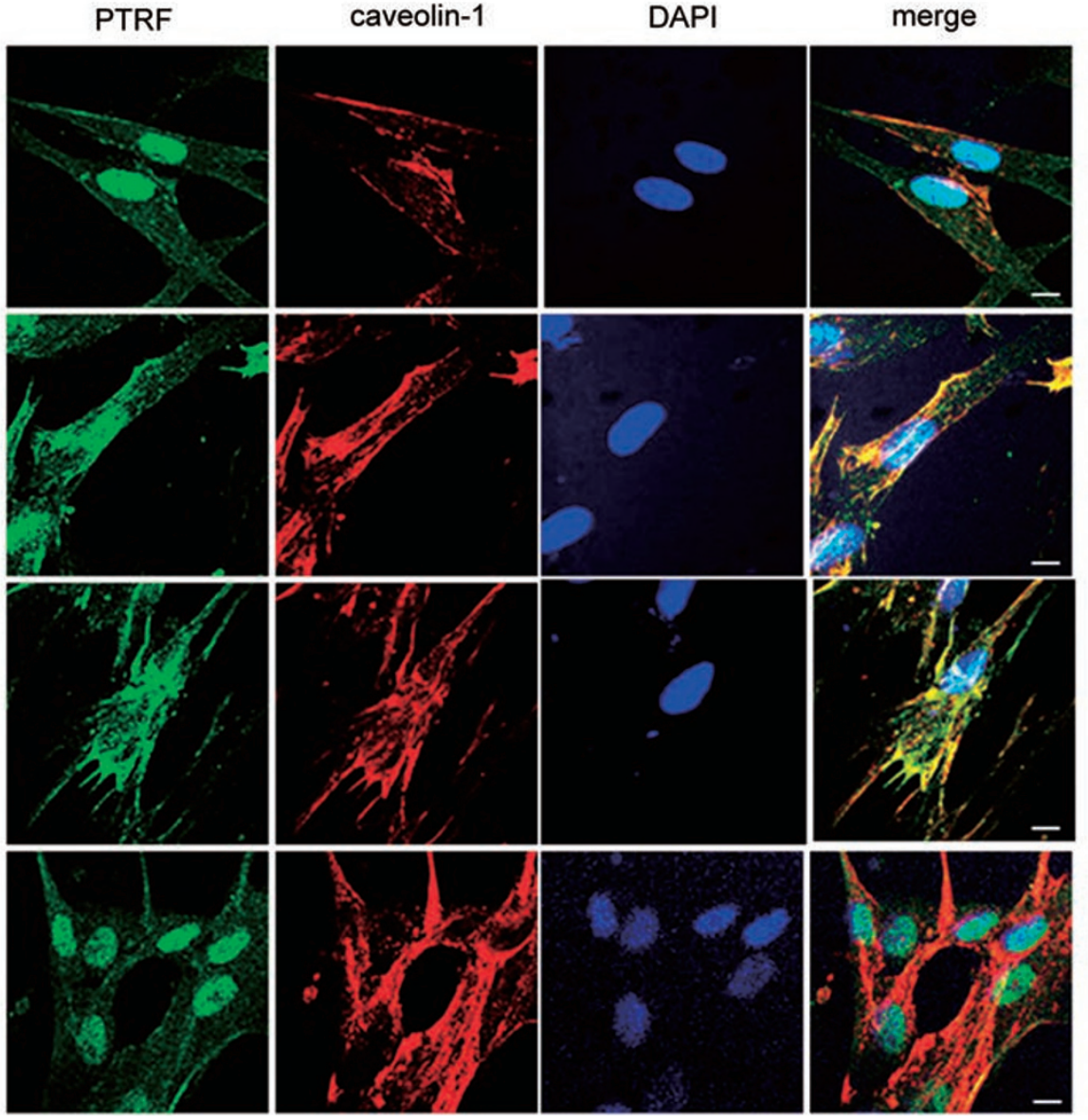

B

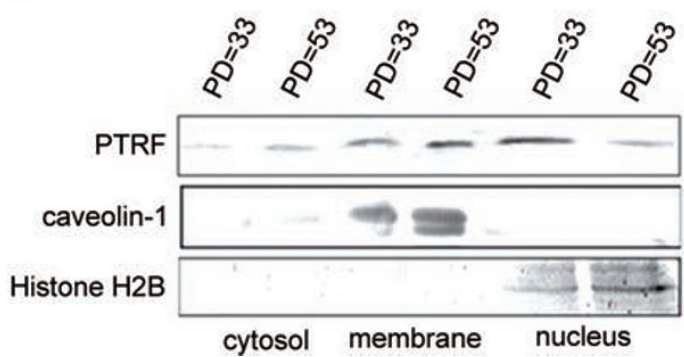

C

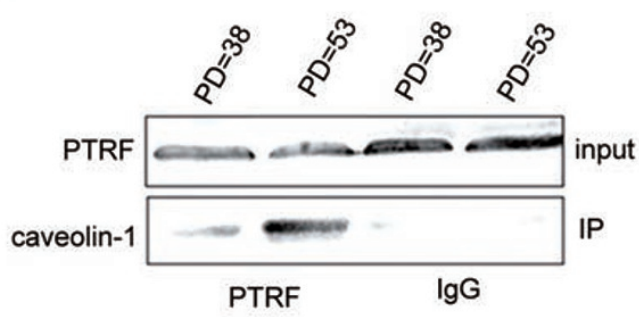

Figure 5 Subcellular distribution of PTRF in WI-38 cells at different growth stages. (A) Subcellular distribution of PTRF in young replicating $(P D=33)$, middle-aged replicating $(P D=43)$, replicatively senescent $(P D=53)$ and young quiescent WI38 fibroblasts. Cells were fixed, stained with antibodies against PTRF (green), caveolin-1 (red) and DAPI (blue), and visualized by laser-scanning confocal microscopy. The scale bar in (A) represents $10 \mu \mathrm{m}$. (B) Subcellular distribution of PTRF detected by western blot analysis of PTRF in cytosolic, membrane and nuclear fractions of young replicating and replicatively senescent WI-38 cells. The expression levels of caveolin-1 and histone H2B were used as markers for membrane and nuclear proteins, respectively. (C) Total cell lysates from young replicating and replicatively senescent WI-38 cells were immunoprecipitated with anti-PTRF and anti-lgG and immune complexes were analyzed by western blot using an anti-caveolin-1 antibody. 
we discovered an increased number of caveolar structures in senescent WI-38 cells compared to young WI38 cells (Figure 6A). In addition, transfection of PTRF expression constructs into young WI-38 cells resulted in an increased number of caveolar structures, further confirming the role of PTRF in the formation of caveolae in human fibroblasts. The size of the caveolae induced by PTRF overexpression, however, was smaller than that observed in replicatively senescent cells (Figure 6B). It is possible that the size of caveolae is regulated by other factors such as SDPR. It has been recently demonstrated that overexpression of SDPR does not significantly alter the total number of caveolae but leads to profuse tubulation of the plasma membrane [21].

Effects of mutations at serine phosphorylation sites of PTRF on its cellular localization

The phosphorylations of PTRF at Ser36, Ser40, Ser365 and Ser366 have been previously reported in human adipocytes by proteomic analysis [19]. In an effort

A
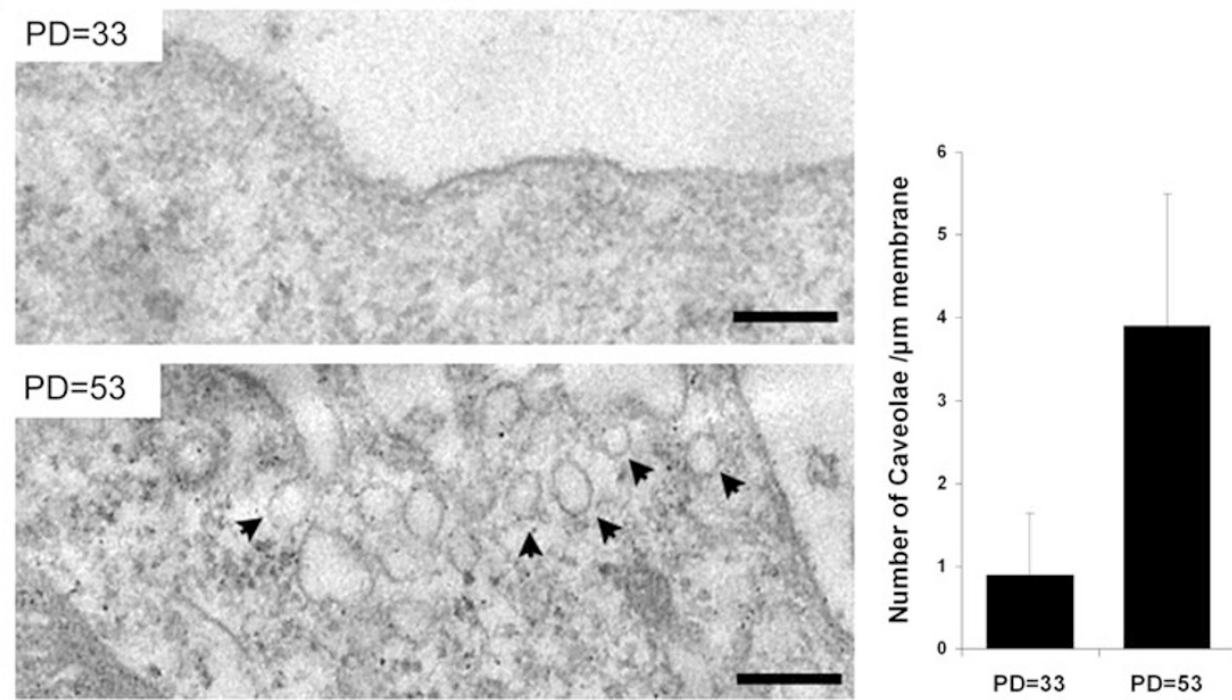

$\mathrm{B}$
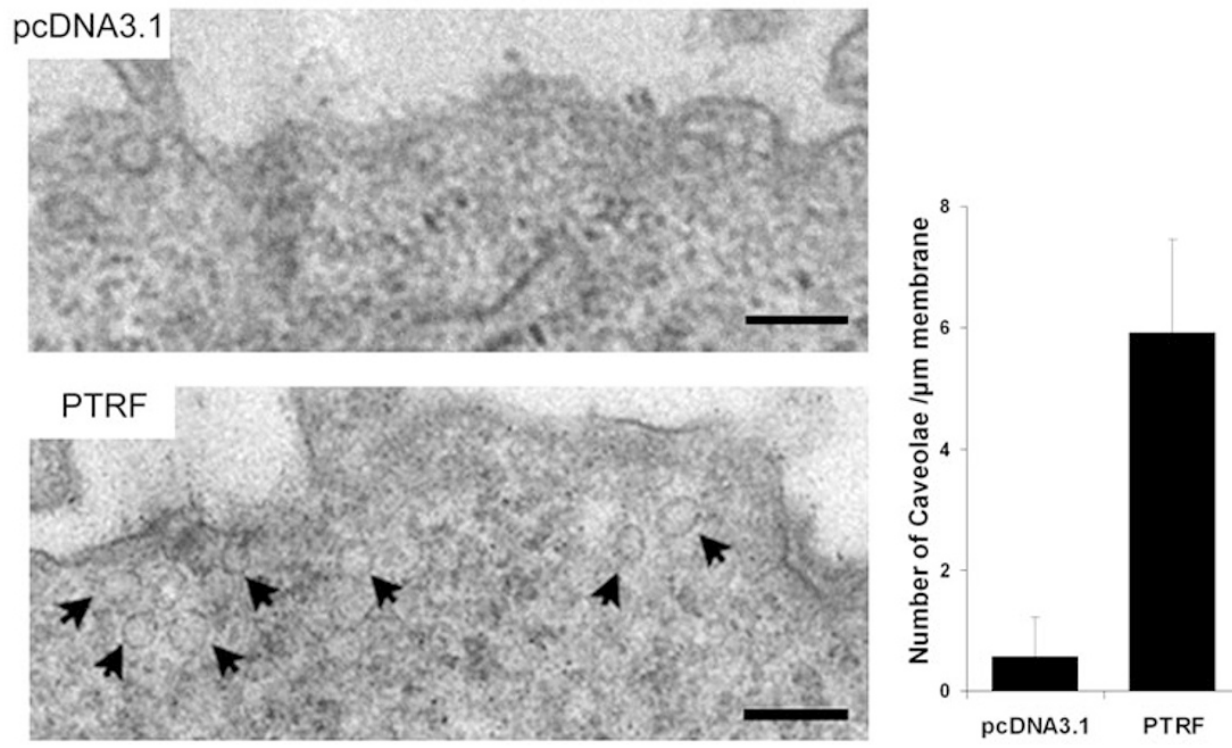

Figure 6 Increased numbers of caveolar structures in senescent and PTRF-transfected cells. (A) Electron micrographs of young $(P D=33)$ and senescent $(P D=53)$ WI-38 cells show an increased number of caveolae during cellular senescence. (B) PTRF-transfected WI-38 cells exhibited an increased number of caveolar structures (indicated by arrows). The number of caveolar structures was statistically analyzed in 10 independent cells. The scale bar represents $200 \mathrm{~nm}$. 
to understand the role of these serine phosphorylation sites in the cellular localization of PTRF, we generated GFP fusion constructs of wild-type PTRF or PTRF mutants in which Ser36, Ser40, Ser365 and Ser366 were replaced with alanine, either individually or in combination, using site-directed mutagenesis (Figure 7A). Wildtype PTRF fused to the amino terminus of GFP displayed nuclear localization in young WI-38 cells but cytosolic and membrane localization in senescent WI-38 cells, similar to endogenous PTRF (data not shown). Transient transfection of the PTRF mutant constructs into young WI-38 cells revealed that in contrast to wild-type PTRFGFP, the S36A, S40A, S36/40A and S36/40/365/366A mutants displayed cytosolic and membrane localization, whereas S365A, S366A, S365/366A mutants retained a nuclear localization (Figure 7B). Western blot analysis using antibodies against GFP showed that there was no degradation of any of these fusion proteins (Supplementary information, Figure S4A), which suggested that the observed cellular localization of these fusion proteins was not due to protein degradation. These results suggest that phosphorylation of PTRF at Ser36 and Ser40, but not at Ser365 and Ser366, is important for its cellular localization and that the subcellular distribution of PTRF may be regulated by phosphorylation.

Role of PTRF in cellular senescence depends on its caveolar targeting and its interaction with caveolin-1

As described above, PTRF is upregulated and translocates to the plasma membrane, where it co-localizes with caveolin-1 during cellular senescence. Thus, we postulated that the role of PTRF in cellular senescence may depend on its caveolar targeting and its interaction with caveolin-1. To test this hypothesis, we stably transfected the wild-type PTRF expression construct; PTRF mutant S36/40A, S365/366A and S36/40/365/366A expression constructs; and the pcDNA3.1 control vector into young WI-38 cells. Western blot analysis indicated that wildtype PTRF and all of the PTRF mutants were expressed at comparable levels (Figure 7E) and did not display degradation due to mutation of serine to alanine at the four phosphorylation sites (see Supplementary information, Figure S4B for western blot detection of wild-type and mutant PTRF expression). All of these stable cell lines were then serially passed to generate growth curves. Similar to wild-type PTRF, the PTRF mutant S36/40A, which displayed cytosolic and membrane localization, rapidly induced cellular senescence, whereas the PTRF mutant S365/366A, which retained nuclear localization, had no effect on the induction of senescence, similar to pcDNA3.1 control vector (Figure 7B-7C). Concomitantly, wild-type PTRF and the PTRF mutant S36/40A induced upregulation of p53 and p21 expression and increased the number of SA- $\beta$-gal-positive cells, whereas the PTRF mutant S365/366A and S36/40/365/366A did not (Figure 6D-6E).

Interestingly, although the PTRF mutant S36/40/ $365 / 366$ A was localized to the cytosol and plasma membrane, it had no effect on the induction of senescence (Figure 7B-7C). To investigate whether the induction of senescence by PTRF was dependent on its interaction with caveolin-1, we transiently transfected FLAGtagged wild-type PTRF and FLAG-tagged PTRF mutant S36/40A, S365/366A and S36/40/365/366A constructs into HeLa cells because of their higher transfection efficiency compared to WI-38 cells. The interaction of PTRF with caveolin-1 was then assessed by co-immunoprecipitation. Figure 7F shows the detection of FLAG-tagged PTRF and mutant PTRF expression using the PTRF antibody, which recognizes both FLAG-tagged and endogenous PTRF in cell lysates. The FLAG immunoprecipitates were analyzed by western blot with the caveolin-1 antibody. FLAG-tagged wild-type PTRF and FLAGtagged PTRF S36/40A co-immunoprecipitated with caveolin-1, but the interaction of FLAG-tagged PTRF S365/366A and S36/40/365/366A with caveolin-1 was significantly reduced (Figure $7 \mathrm{~F}$ ). This result suggests that the phosphorylation of PTRF at Ser365 and Ser366 is required for the interaction of PTRF with caveolin-1, consistent with the observation that wild-type PTRF and mutant PTRF S36/40A were both able to induce senescence in WI-38 cells, whereas PTRF S365/366A and S36/40/365/366A were not (Figure 7D).

The role of caveolin-1 in PTRF-induced cellular senescence was further investigated using caveolin-1 RNA interference. Young WI-38 cells were either co-transfected with pcDNA3.1-PTRF and sicaveolin-1 or control siRNA, or co-transfected with the pcDNA3.1 vector and sicaveolin-1 or control siRNA. Consistent with an earlier report showing that downregulation of caveolin-1 reverses senescence [35], transfection of sicaveolin-1, but not control siRNA, resulted in an extended lifespan of WI-38/pcDNA3.1 cells (Figure 8A-8C). Significantly, transfection with sicaveolin-1, but not control siRNA, extended the lifespan of WI-38/pcDNA3.1-PTRF cells, in addition to downregulating p21 and p53 expression and decreasing the number of SA- $\beta$-gal-positive cells (Figure 8A-8C). These results suggest that PTRF regulates cellular senescence in association with caveolin-1.

\section{Discussion}

Our data show that during cellular senescence, PTRF is upregulated and translocates to plasma membrane, 

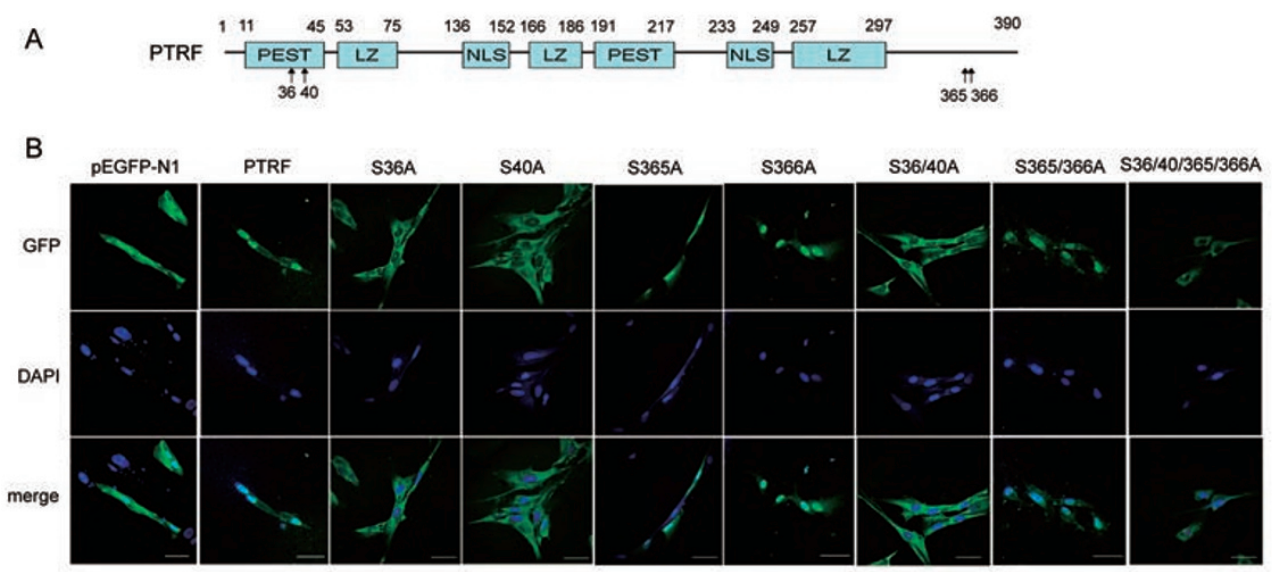

C

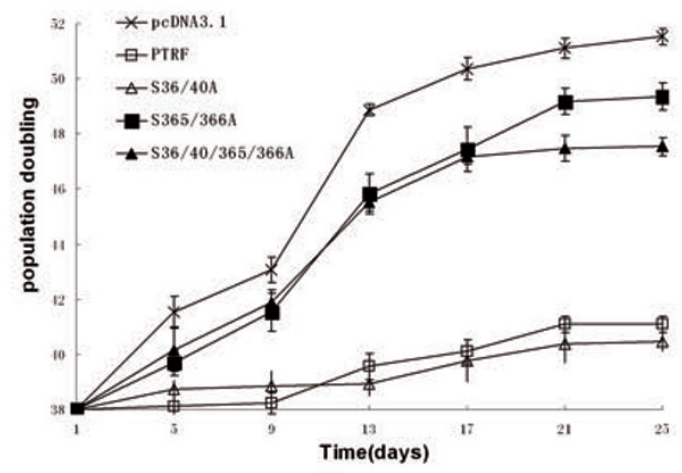

E
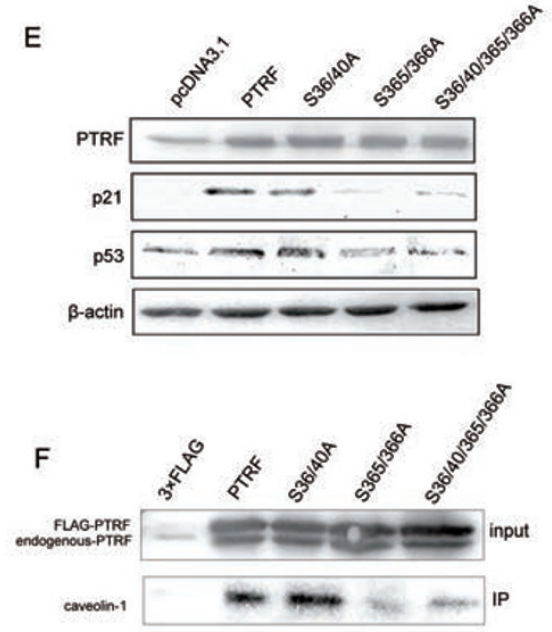

D
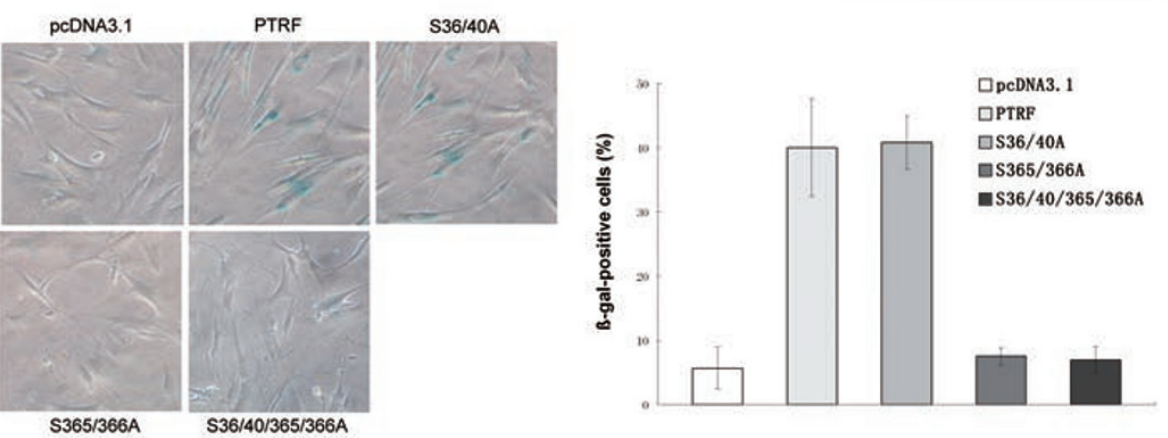

Figure 7 Analysis of serine phosphorylation sites on PTRF with respect to the subcellular distribution of PTRF and cellular senescence. (A) Schematic representation of the domain structure of PTRF protein: putative PEST domain (PEST), leucine zipper domain (LZ), nuclear localization signal (NLS). The numbers correspond to the first and last amino acids of each domain in the protein sequence. The previously identified phosphorylation sites, Ser36, Ser40, Ser365 and Ser366, are indicated by arrows [19]. (B) WI-38 cells transfected with the indicated constructs were fixed and stained with DAPI to stain the nuclei. Cellular localization of wild-type pEGFP-N1-PTRF and the pEGFP-N1-PTRF mutants was determined by confocal microscopy. The scale bar represents $50 \mu \mathrm{m}$. (C) Growth curves of WI38 cells that were stably transfected with the indicated expression constructs. The data represent the means \pm s.d of three independent experiments. (D) SA- $\beta$-gal staining of the cells indicated in (C). The percentage of SA- $\beta$-gal-positive cells was calculated from three randomly chosen fields. The experiment was repeated three times, and the results represent the mean \pm S.d. (E) Western blot analysis of PTRF and the senescence-associated proteins $\mathrm{p} 53$ and p21 in WI-38 cells transfected with the indicated expression constructs. (F) The interaction of PTRF with caveolin-1 depends on the phosphorylation of PTRF at Ser365 and Ser366. HeLa cells were transiently transfected with the indicated constructs, and total cell lysates were subjected to western blot using anti-PTRF (input) or to immunoprecipitation with anti-FLAG antibody. Co-immunoprecipitated caveolin-1 was detected by western blot with anticaveolin-1. 
A

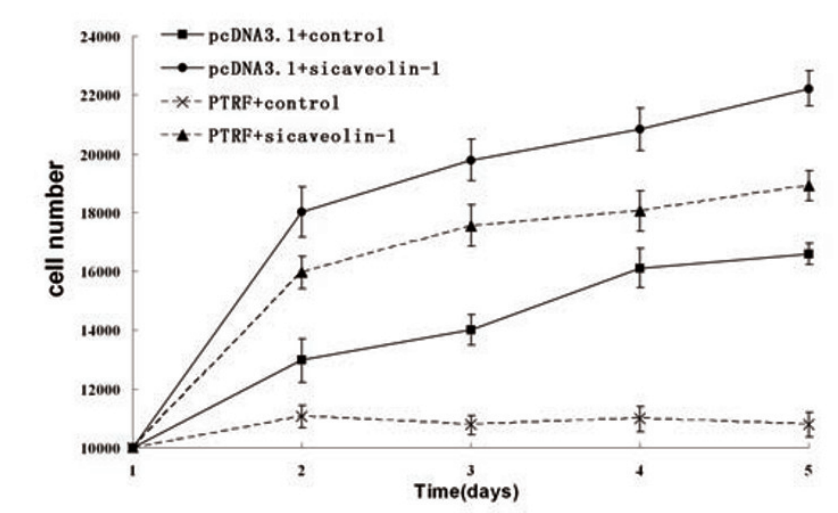

C

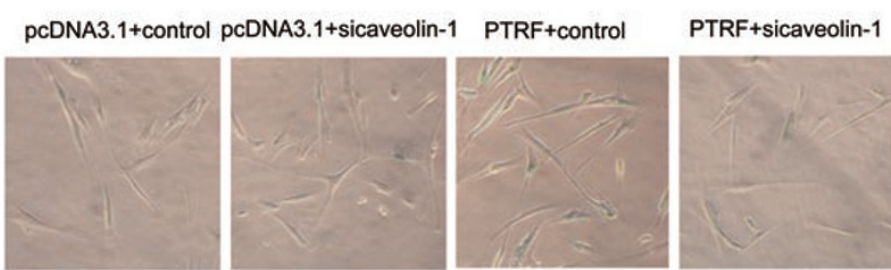

B
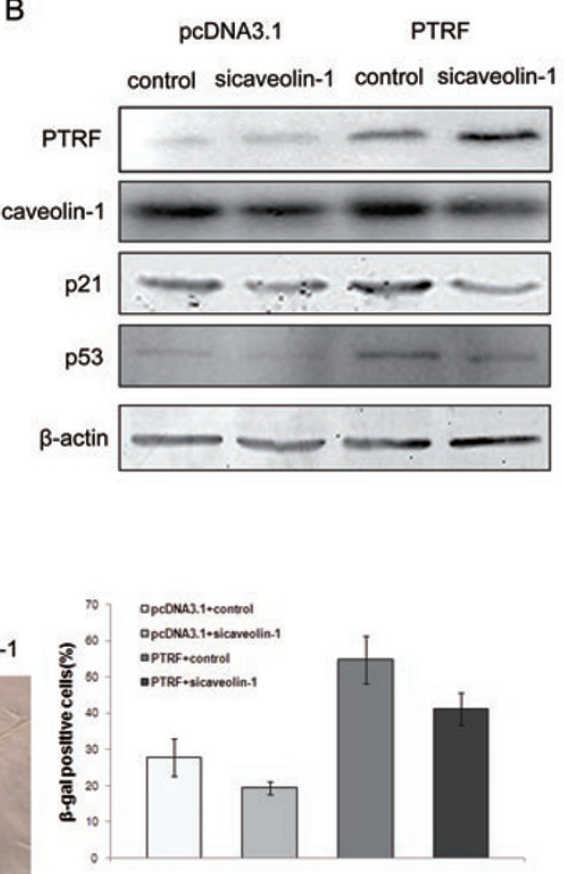

Figure 8 Caveolin-1 is involved in the process of PTRF-induced senescence. (A) WI-38 cells were co-transfected with the indicated constructs and siRNAs. Growth curves of these cell lines were generated by cell number at the indicated times. The data presented are from three independent experiments. (B) Western blot analysis of PTRF, caveolin-1, p53 and p21 expression in the cell lines in (A). (C) SA- $\beta$-gal staining of the cells indicated in (A). The percentage of SA- $\beta$-gal-positive cells was calculated from three randomly chosen fields. The experiment was repeated three times, and the results represent as the mean \pm s.d.

where it interacts with caveolin-1 and promotes the formation of caveolae. Overexpression of PTRF induced phenotypes characteristic of cellular senescence, whereas knockdown of PTRF by shRNA resulted in the opposite phenotypes and an extended cellular lifespan. We subsequently showed that the effects of PTRF on cellular senescence were associated with key regulators of senescence, including p53 and p21, and known signaling molecules of the caveolar pathway, such as ERK1/2 and cyclin D1. These results suggest that PTRF regulates cellular senescence by modulating caveolar/p53/p21 pathways.

Accumulation of $\gamma-\mathrm{H} 2 \mathrm{~A} . \mathrm{X}$ foci is a common process in mammalian aging in vivo and in culture. The $\gamma$-H2A.X signals randomly co-localize with the telomere signals in aging mice [36], and in replicatively senescent human fibroblasts [37]. PTRF-induced senescence was associated with accumulation of the $\gamma$-H2A.X foci, although the etiology of the foci have not been investigated in this study; however, since our results showed that PTRF-induced senescence was independent of telomeres, the foci we observed may not specifically at telomeres. During senescence, senescence-associated heterochromatic foci are formed by global chromatin structural changes, resulting in widespread epigenetic changes in gene expression and silencing of cell cycle genes. PTRF may induce premature senescence by activation of caveolae/p53/p21 pathways, and consequently accumulation of DNA foci containing $\gamma$-H2A.X.

Previous studies show that $\mathrm{p} 21$ and $\mathrm{p} 16$ are upregulated independently in senescence [38]. Similarly, the insulin-like growth factor binding protein-5 induces cellular senescence dependent on the p53/p21 pathway but not p16 [39]. We showed that PTRF was upregulated in senescent cells and promote caveolae formation, which leads to increased caveolin-1, p53 and p21, suggesting that PTRF modulates p53 stability through the caveolae/caveolin-1 pathway. In addition, we found that knockdown p53 expression attenuated PTRF-induced senescence (Supplementary information, Figure S5), indicating that the ability of PTRF to induce senescence requires $\mathrm{p} 53$. In agreement with our observation, a recent study demonstrates that caveolin-1 is a novel binding protein for Mdm2. After oxidative stress, caveolin-1 sequesters Mdm2 away from p53, leading to stabilization of p53 and upregulation of p21 in human fibroblasts [40]. 
Take together, these results suggest that PTRF regulates cellular senescence by modulating the caveolae/p53/p21 pathway.

It has been proposed that a network of kinases regulates caveolar functions [41, 42]. A number of phosphorylation sites have been identified in PTRF in different cell types [32], which suggests that the phosphorylation may be an important mechanism in the post-translational modification of PTRF during cellular senescence. Using site-directed mutagenesis analysis, we analyzed the four previously identified phosphorylation sites of PTRF (Ser36, Ser40, Ser365 and Ser366) [19] and found that they were important for the cellular localization of PTRF and its interaction with caveolin-1. Furthermore, the role of PTRF in cellular senescence was dependent not only on its caveolar localization but also on its interaction with caveolin-1.

Human PTRF mutations have been identified in patients with generalized lipodystrophy [26, 27], and analysis of skeletal muscle biopsies from these patients has revealed a deficiency and mislocalization of all three caveolin family members and a reduction in the number of caveolar structures [27]. One of these PTRF mutations with a C-terminal deletion encompassing the Ser356 and Ser366 phosphorylation sites analyzed in this study displays a nuclear and cytoplasmic localization [27]. This finding is consistent with our data showing that PTRF mutants S365A and S366A retain a nuclear localization, fail to interact with caveolin-1, and are deficient in the induction of senescence.

Caveolin family proteins are the best-studied components of caveolae [18]. Caveolin-1 has been shown to regulate cellular senescence $[40,43,44]$ in a manner similar to PTRF in the present study. PTRF is an essential component in biogenesis and function of caveolae $[24,30]$. Recently, studies have demonstrated that both SDPR and SRBC are required for caveolar formation [20, 21]. PTRF, SDPR, SRBC and caveolin-1 appear to form a protein complex in which the expression of one member may affect both the expression of other members and caveolar dynamics [21, 22, 45]. The underlying mechanisms for this regulation, however, are currently unknown. Several questions remain unanswered by the present study, including how PTRF is regulated by phosphorylation and how PTRF regulates caveolar signaling during cellular senescence. A complete understanding of the role of caveolae in cellular senescence requires studies in the context of the emerging picture of caveolar composition/signaling and dynamic regulation. Understanding the mechanisms of caveolar regulation by PTRF will provide molecular insights into the roles of caveolar signaling in aging.

\section{Materials and Methods}

\section{Antibodies}

The following antibodies were used: anti- $\beta$-actin (13E5), anti$\mathrm{Rb}$ (4H1), anti-p53, anti-p21 ${ }^{\text {Wafl/Cip1 }}$ (12D1), anti-phospho-p44/42 MAP kinase (Thr202/Tyr204), and anti-p44/42 MAP kinase were from Cell Signaling (Beverly, MA); antibodies against PTRF and caveolin-1 were from BD Pharmingen (San Jose, CA); anticollagen Type I was from US Biological (Swampscott, MA); antiHSP90 and anti-hTERT were from Abcam (Cambridge, UK); anti$\gamma$-H2A.X (ser139) was from Upstate Biotechnology (Lake Placid, $\mathrm{NY}$ ); and anti-histone H2B and anti-cyclin D1 were from Santa Cruz Biotechnology (Santa Cruz, CA).

\section{Cell culture and growth curves}

WI-38, IMR-90 human diploid fibroblasts and HeLa cells were cultured in Dulbecco's modified Eagle's medium containing 10\% fetal bovine serum, $1 \%$ L-glutamine and 1\% penicillin/streptomycin at $37{ }^{\circ} \mathrm{C}$ and $5 \% \mathrm{CO}_{2}$. Quiescent, replicatively senescent and prematurely senescent WI-38 populations were prepared as described previously [28]. For the cell proliferation experiments, cells were seeded in 24-well plates in triplicate, and the cell number was counted by using a Beckman Coulter $\mathrm{Z} 1$ cell counter. $\mathrm{Cu}$ mulative population doubling (PD) was determined as described previously [46].

\section{Plasmids, siRNAs and transfection}

A full-length cDNA of PTRF was obtained by RT-PCR using specific primers (forward: 5'-ATGGAGGACCCCACGCTCTATA -3'; reverse: 5'-CCGAGAGAGAATGCGAAAGAGG-3') with total RNA from WI-38 cells. The PTRF cDNA was cloned into the pGEM-T-easy vector and subcloned into pcDNA3.1, p3XFLAG-CMV and pEGFP-N1. For the caveolin-1 knockdown experiments, a mixture of two siRNAs against two targets (sense 5'-AAGGAGATCGACCTGGTCAAC-3' and 5'-AAGGGACACACAGTTTTGACG) as described in [47], and non-specific control siRNA duplexes (sense 5'-TTCTCCGAACGTGTCACGT-3') were purchased from Shanghai Genepharma Co., Ltd (China). Transfections were performed using Lipofectamine 2000 according to the manufacturer's protocol. Drug selection of the cells was performed with G418 (0.9 $\mu \mathrm{g} / \mathrm{ml}$ for WI-38) for 5-7 days.

\section{shRNA constructs and retroviral infection}

For the PTRF shRNA constructs, the hairpin sequences were as follows: the shPTRF1 sequences spanned nucleotides $1762-$ 1780 of PTRF (forward, 5'-tcgagcgGGAAAGATTGAATCCTAAAttgatatccgTTTAGGATTCAATCTTTCCttttttccaaag-3', and reverse, 5'-aattcttggaaaaaaGGAAAGATTGAATCCTAAAcggatatcaaTTTAGGATTCAATCTTTCCegc-3'), and the shPTRF2 sequence spanned nucleotides 3 096-3 114 of PTRF (forward, 5' -tcgagcgGACACGACCAGGTTCTCAAttgatatccgTTGAGAACCTGGTCGTGTCttttttccaaag-3', and reverse, 5'-aattcttggaaaaaGACACGACCAGGTTCTCAAcggatatcaaTTGAGAACCTGGTCGTGTCcgc-3'). The negative control shRNA had no significant homology to any mammalian gene sequences, and its sequence was as follows: forward, 5'-tcgagcgTTCTCCGAACGTGTCACGTttgatatccgACGTGACACGTTCGGAGAAtttttccaaag-3', and reverse, 5'-aattcttggaaaaaaTCTCCGAACGTGTCACGTcggatatcaaACGTGACACGTTCGGAGAAcge-3'. These 
shRNA sequences were cloned into the MSCV-TMP vector and confirmed by DNA sequencing.

Retroviral supernatants were produced by PA317 packaging cells that were stably transfected with the retroviral constructs and filtered through a $45 \mu \mathrm{m}$ Millex HA filter (Millipore). Retroviral infections were performed in the presence of $4 \mu \mathrm{g} / \mathrm{ml}$ polybrene (Sigma). Drug selection of the cells was performed with puromycin $(1 \mu \mathrm{g} / \mathrm{ml}$ for WI-38, $2 \mu \mathrm{g} / \mathrm{ml}$ for PA317) or G418 $(0.9 \mu \mathrm{g} / \mathrm{ml}$ for WI-38) for 5-7 days.

\section{Western blot analysis and immunoprecipitation}

For immunoblotting, cell lysates were prepared in RIPA buffer (50 mM Tris. $\mathrm{Cl}$, pH 8.0, $100 \mathrm{mM} \mathrm{NaCl}, 0.1 \%$ SDS, $0.5 \%$ sodium deoxycholate, $1 \% \mathrm{NP}-40$, and a protease inhibitor cocktail (Roche)). Total protein concentrations were measured using BCA kit, and immunoblotting was performed with a clarified extract containing 20-60 $\mu \mathrm{g}$ total protein that was separated on $12 \%$ SDSpolyacrylamide gels. Proteins were transferred to PVDF membrane (Millipore) and incubated at $4{ }^{\circ} \mathrm{C}$ overnight with primary antibodies. Alkaline phosphatase-conjugated anti-mouse or antirabbit secondary antibodies were used for detection using a BCIP/ NBT color development substrate (Promega). Subcellular fractions of cells were prepared using a subcellular proteome extraction kit (Calbiochem) according to the manufacturer's protocol. For immunoprecipitation, cells were lysed in NP-40 buffer $(50 \mathrm{mM}$ Tris $\cdot \mathrm{Cl}$, $\mathrm{pH} 7.4,150 \mathrm{mM} \mathrm{NaCl}, 0.5 \%$ sodium deoxycholate, 1\% NP-40 and protease inhibitor cocktail). Clarified extract was incubated with 20-30 $\mu 1$ M2 anti-FLAG resin (Sigma) for $1-2 \mathrm{~h}$ at $4{ }^{\circ} \mathrm{C}$. The associated proteins were analyzed by immunoblotting.

\section{Immunofluorescence}

Cells were plated in six-well plates with glass coverslips, fixed with $4 \%$ paraformaldehyde in phosphate buffered saline (PBS), and permeabilized in $0.5 \%$ Triton X-100. After blocking of nonspecific antigens with $5 \%$ bovine serum albumin, hybridization with the primary antibody at a 1:50 dilution was performed at $37^{\circ} \mathrm{C}$ for $1 \mathrm{~h}$. Hybridization with a FITC-conjugated or TRITC-conjugated secondary antibody was performed at $37^{\circ} \mathrm{C}$ for $30 \mathrm{~min}$, and then the cells were stained with DAPI $(1 \mu \mathrm{g} / \mathrm{ml})$ for $5 \mathrm{~min}$. Imaging was performed using an Olympus FluoView confocal laser scanning system, and images were captured using Olympus FluoView (FV300) software (Tokyo, Japan).

\section{$R T-P C R$}

Total RNA was extracted from WI-38 and IMR-90 cells using TRIzol reagent (Invitrogen, Paisley, UK) according to the manufacturer's instructions. Genes of interest were amplified from 2 $\mu \mathrm{g}$ of DNase I-treated total RNAs using M-MLV Reverse Transcriptase (Promega, Madison, USA) and poly dT primer.

The primers used for PCR were as follows: PTRF (forward: 5'-ACGCCACCACGAGCAATAC-3'; reverse: 5'-CGGCAGCTTCACTTCATCC-3', $T_{\mathrm{m}}=60^{\circ} \mathrm{C}, 26$ cycles), and $G A P D H$ (forward: 5'-TGCTAAGCAGTTGGTGGTGCAGGA-3'; reverse: 5'-CGGAGTCAACGGATTTGGTCGTAT- $3^{\prime} ; T_{\mathrm{m}}=57^{\circ} \mathrm{C}, 22$ cycles). After amplification, the PCR products were separated on $1.5 \%$ agarose gels and visualized by ethidium bromide staining.

\section{$S A-\beta$-gal staining}

The SA- $\beta$-gal assay was performed as described previously [6]. Briefly, the cells were washed with PBS and fixed with $0.5 \%$ glutaraldehyde in PBS for $5 \mathrm{~min}$ at room temperature. After washing with PBS, the cells were incubated with a freshly prepared staining solution ( $1 \mathrm{mg} / \mathrm{ml}$ 5-bromo-4-chloro-3-indolyl- $\beta$-Dgalactopyranoside (X-gal), $40 \mathrm{mM}$ citric acid/sodium phosphate, $\mathrm{pH}$ 6.0, $5 \mathrm{mM}$ potassium ferrocyanide, $5 \mathrm{mM}$ potassium ferricyanide, $15 \mathrm{mM} \mathrm{NaCl}$ and $2 \mathrm{mM} \mathrm{MgCl}_{2}$ ) at $37{ }^{\circ} \mathrm{C}$ for $16 \mathrm{~h}$.

\section{Electron microscopic analysis}

Subconfluent young replicating and senescent WI-38 cells were pelleted and fixed with $3 \%$ glutaraldehyde in PBS at $\mathrm{pH} 7.4$. The cell pellets were washed with PBS and treated with $1 \%$ osmium tetroxide in PBS buffer for $1 \mathrm{~h}$. After washing again with PBS, the cells were dehydrated in graded acetone and embedded in epoxy resin at $37{ }^{\circ} \mathrm{C}$ for $12 \mathrm{~h}$ and at $60{ }^{\circ} \mathrm{C}$ for $48 \mathrm{~h}$. Ultrathin sections were cut and stained with uranyl acetate and lead citrate. The sections were then observed using a transmission electron microscope (JSM-1230).

\section{Acknowledgments}

We thank Dr Silvia Bacchetti for critical reading of the manuscript, Dr Pingsheng Liu for providing the caveolin-1 siRNA and helpful discussions. This work was supported in part by a grant from the National Natural Science Foundation of China (30671065) and grants from the National Basic Research Program of China (973 Program) (2007CB507402, 2007CB914402).

\section{References}

1 Hayflick L. The limited in vitro lifetime of human diploid cell strains. Exp Cell Res 1965; 37:614-636.

2 Campisi J, d'Adda di Fagagna F. Cellular senescence: when bad things happen to good cells. Nat Rev Mol Cell Biol 2007; 8:729-740.

3 Ben-Porath I, Weinberg RA. When cells get stressed: an integrative view of cellular senescence. J Clin Invest 2004; 113:813.

4 Campisi J. Senescent cells, tumor suppression, and organismal aging: good citizens, bad neighbors. Cell 2005; 120:513522.

5 Hayflick L. Theories of biological aging. Exp Gerontol 1985; 20:145-159.

6 Dimri GP, Lee X, Basile G, et al. A biomarker that identifies senescent human cells in culture and in aging skin in vivo. Proc Natl Acad Sci USA 1995; 92:9363-9367.

7 Jeyapalan JC, Sedivy JM. Cellular senescence and organismal aging. Mech Ageing Dev 2008; 129:467-474.

8 Campisi J. Cellular senescence as a tumor-suppressor mechanism. Trends Cell Biol 2001; 11:S27-S31.

9 Wright WE, Shay JW. Cellular senescence as a tumor-protection mechanism: the essential role of counting. Curr Opin Genet Dev 2001; 11:98-103.

10 Braig M, Lee S, Loddenkemper C, et al. Oncogene-induced senescence as an initial barrier in lymphoma development. Nature 2005; 436:660-665.

11 Chen Z, Trotman LC, Shaffer D, et al. Crucial role of p53dependent cellular senescence in suppression of Pten-deficient tumorigenesis. Nature 2005; 436:725-730.

12 Collado M, Serrano M. The senescent side of tumor suppression. Cell Cycle 2005; 4:1722-1724. 
13 Michaloglou C, Vredeveld LC, Soengas MS, et al. BRAFE600-associated senescence-like cell cycle arrest of human naevi. Nature 2005; 436:720-724.

14 Ventura A, Kirsch DG, McLaughlin ME, et al. Restoration of p53 function leads to tumour regression in vivo. Nature 2007; 445:661-665.

15 Xue W, Zender L, Miething C, et al. Senescence and tumour clearance is triggered by $\mathrm{p} 53$ restoration in murine liver carcinomas. Nature 2007; 445:656-660.

16 Martins CP, Brown-Swigart L, Evan GI. Modeling the therapeutic efficacy of p53 restoration in tumors. Cell 2006; 127:1323-1334.

17 Finkel T, Serrano M, Blasco MA. The common biology of cancer and ageing. Nature 2007; 448:767-774.

18 Parton RG, Simons K. The multiple faces of caveolae. Nat Rev Mol Cell Biol 2007; 8:185-194.

19 Aboulaich N, Vainonen JP, Stralfors P, Vener AV. Vectorial proteomics reveal targeting, phosphorylation and specific fragmentation of polymerase I and transcript release factor (PTRF) at the surface of caveolae in human adipocytes. Biochem J 2004; 383:237-248.

20 McMahon KA, Zajicek H, Li WP, et al. SRBC/cavin-3 is a caveolin adapter protein that regulates caveolae function. EMBO J 2009; 28:1001-1015.

21 Hansen CG, Bright NA, Howard G, Nichols BJ. SDPR induces membrane curvature and functions in the formation of caveolae. Nat Cell Biol 2009; 11:807-814.

22 Bastiani M, Liu L, Hill MM, et al. MURC/Cavin-4 and cavin family members form tissue-specific caveolar complexes. $J$ Cell Biol 2009; 185:1259-1273.

23 Jansa P, Mason SW, Hoffmann-Rohrer U, Grummt I. Cloning and functional characterization of PTRF, a novel protein which induces dissociation of paused ternary transcription complexes. EMBO J 1998; 17:2855-2864.

24 Hill MM, Bastiani M, Luetterforst R, et al. PTRF-Cavin, a conserved cytoplasmic protein required for caveola formation and function. Cell 2008; 132:113-124.

25 Liu L, Brown D, McKee M, et al. Deletion of Cavin/PTRF causes global loss of caveolae, dyslipidemia, and glucose intolerance. Cell Metab 2008; 8:310-317.

26 Rajab A, Straub V, McCann LJ, et al. Fatal cardiac arrhythmia and long-QT syndrome in a new form of congenital generalized lipodystrophy with muscle rippling (CGL4) due to PTRF-CAVIN mutations. PLoS Genet 2010; 6:e1000874.

27 Hayashi YK, Matsuda C, Ogawa M, et al. Human PTRF mutations cause secondary deficiency of caveolins resulting in muscular dystrophy with generalized lipodystrophy. J Clin Invest 2009; 119:2623-2633.

28 Cong YS, Fan E, Wang E. Simultaneous proteomic profiling of four different growth states of human fibroblasts, using amine-reactive isobaric tagging reagents and tandem mass spectrometry. Mech Ageing Dev 2006; 127:332-343.

29 Park WY, Park JS, Cho KA, et al. Up-regulation of caveolin attenuates epidermal growth factor signaling in senescent cells. J Biol Chem 2000; 275:20847-20852.

30 Liu L, Pilch PF. A critical role of cavin (polymerase I and transcript release factor) in caveolae formation and organization. J Biol Chem 2008; 283:4314-4322.

31 Liu P, Rudick M, Anderson RG. Multiple functions of caveo- lin-1. J Biol Chem 2002; 277:41295-41298.

32 Hansen CG, Nichols BJ. Exploring the caves: cavins, caveolins and caveolae. Trends Cell Biol 2010; 20:177-186.

33 Bodnar AG, Ouellette M, Frolkis M, et al. Extension of lifespan by introduction of telomerase into normal human cells. Science 1998; 279:349-352.

34 Zhou L, Zheng D, Wang M, Cong YS. Telomerase reverse transcriptase activates the expression of vascular endothelial growth factor independent of telomerase activity. Biochem Biophys Res Commun 2009; 386:739-743.

35 Cho KA, Ryu SJ, Park JS, et al. Senescent phenotype can be reversed by reduction of caveolin status. J Biol Chem 2003; 278:27789-27795.

36 Wang C, Jurk D, Maddick M, et al. DNA damage response and cellular senescence in tissues of aging mice. Aging Cell 2009; 8:311-323.

37 Sedelnikova OA, Horikawa I, Zimonjic DB, Popescu NC, Bonner WM, Barrett JC. Senescing human cells and ageing mice accumulate DNA lesions with unrepairable doublestrand breaks. Nat Cell Biol 2004; 6:168-170.

38 Herbig U, Jobling WA, Chen BP, Chen DJ, Sedivy JM. Telomere shortening triggers senescence of human cells through a pathway involving ATM, p53, and p21(CIP1), but not p16(INK4a). Mol Cell 2004; 14:501-513.

39 Kim KS, Seu YB, Baek SH, et al. Induction of cellular senescence by insulin-like growth factor binding protein-5 through a p53-dependent mechanism. Mol Biol Cell 2007; 18:45434552 .

40 Bartholomew JN, Volonte D, Galbiati F. Caveolin-1 regulates the antagonistic pleiotropic properties of cellular senescence through a novel Mdm2/p53-mediated pathway. Cancer Res 2009; 69:2878-2886.

41 Pelkmans L, Fava E, Grabner H, et al. Genome-wide analysis of human kinases in clathrin- and caveolae/raft-mediated endocytosis. Nature 2005; 436:78-86.

42 Pelkmans L, Zerial M. Kinase-regulated quantal assemblies and kiss-and-run recycling of caveolae. Nature 2005; 436:128-133.

43 Dasari A, Bartholomew JN, Volonte D, Galbiati F. Oxidative stress induces premature senescence by stimulating caveolin-1 gene transcription through p38 mitogen-activated protein kinase/Sp1-mediated activation of two GC-rich promoter elements. Cancer Res 2006; 66:10805-10814.

44 Volonte D, Kahkonen B, Shapiro S, Di Y, Galbiati F. Caveolin-1 expression is required for the development of pulmonary emphysema through activation of the ATM-p53-p21 pathway. J Biol Chem 2009; 284:5462-5466.

45 Nabi IR. Cavin fever: regulating caveolae. Nat Cell Biol 2009; 11:789-791.

46 Shay JW, Wright WE. Quantitation of the frequency of immortalization of normal human diploid fibroblasts by SV40 large T-antigen. Exp Cell Res 1989; 184:109-118.

47 Vallejo J, Hardin CD. Caveolin-1 functions as a scaffolding protein for phosphofructokinase in the metabolic organization of vascular smooth muscle. Biochemistry 2004; 43:1622416232.

(Supplementary information is linked to the online version of the paper on the Cell Research website.) 